\title{
Polymeric capsules and honeycomb aggregates formed by $p$ - sulfonatocalix[6]arene with phenanthrolinium compounds
}

\section{Yu Liu*, Qiang Li, Dong-Sheng Guo and Kun Chen}

Department of Chemistry, State Key Laboratory of Elemento-Organic Chemistry, Nankai University, Tianjin 300071, P. R. China.

Tel: 86-22-23503625, Fax: 86-22-23503625, E-mail: yuliu@ nankai.edu.cn

\section{Powder X-ray diffraction Analysis}

Power X-ray diffraction (PXRD) patterns were obtained using a Rigaku D/max 2500 diffractometer with $\mathrm{Cu} \mathrm{K} \alpha$ radiation $(40 \mathrm{kV}, 100 \mathrm{~mA})$. The PXRD patterns of the two complexes are shown in Figure S1. These patterns are consistent with those of simulated based on the structure models derived from the $\mathbf{X}$-ray crystal structure analysis data, suggesting the phase purity of the complexes.
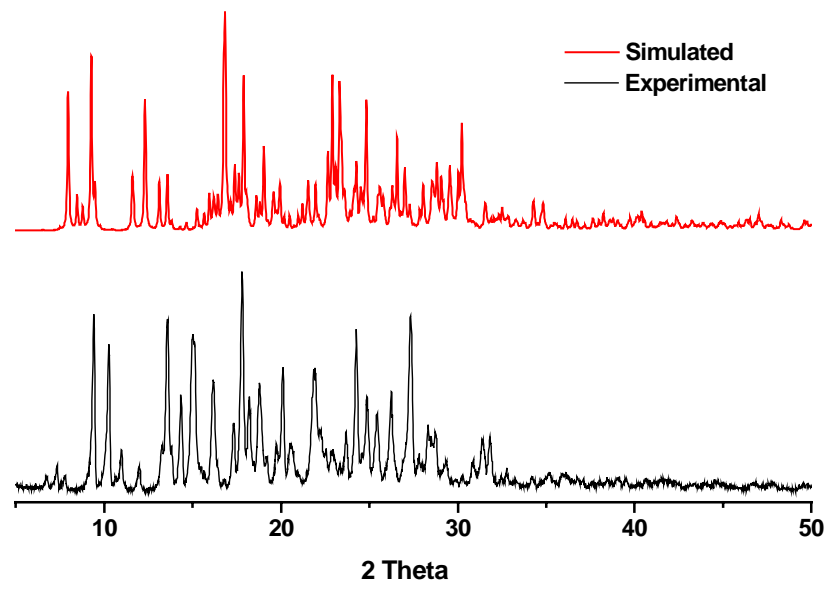

$\mathbf{a}$
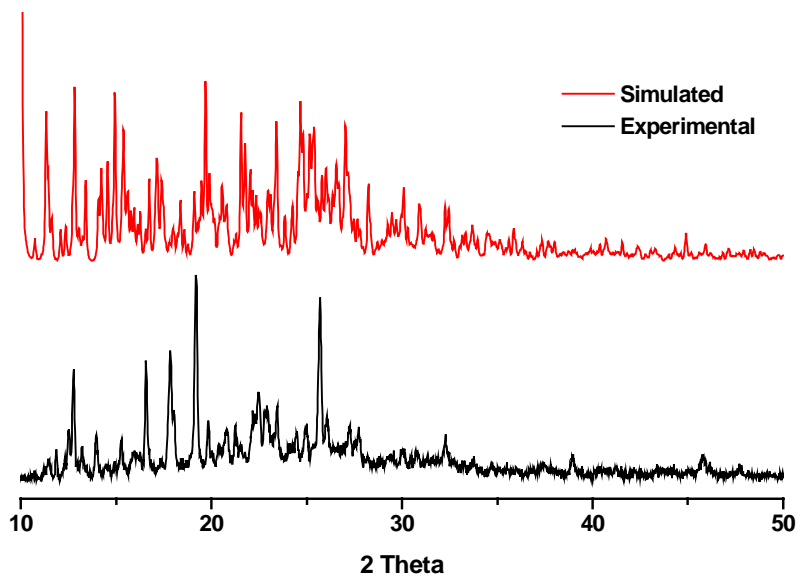

b 
Figure S1. Comparison of PXRD patterns for complex 1 (a) and 2 (b): the top and the bottom patterns correspond to simulated and experimental results, respectively. 